\title{
THE GEOMETRY OF IMMERSIONS. II
}

\author{
BY E. A. FELDMAN ${ }^{1}$ \\ Communicated by J. W. Milnor, February 10, 1964
}

This announcement is the sequel to the announcement Geometry of immersions. I, which appeared in the September 1963 issue of this journal. In the present announcement we develop the characteristic class theory of Thom (see [6], [7]) to include some situations of differential geometric interest. We then apply these techniques of differential topology to some problems of extrinsic differential geometry. Most notable among them is $\$ 6$ which deals with the problem of counting umbilic points on an immersed hypersurface.

In this announcement we draw freely on the definitions contained in our first announcement. Full details will appear in a separate publication.

1. $G$-structures on vector bundles. Their singularities and characteristic classes.

Definition 1.1. Let $\phi_{1}: G \rightarrow \mathrm{GL}(p, R)$ and $\phi_{2}: H \rightarrow \mathrm{GL}(q, R)$ be faithful smooth representations of the Lie groups $G$ and $H$ in $\mathrm{GL}(p, R)$ and $\operatorname{GL}(q, R)$ respectively. This induces in a natural way an action of $G \times H$ on $\operatorname{Hom}\left(R^{p}, R^{q}\right)$. Let $K$ be a regular manifold collection of submanifolds of $\operatorname{Hom}\left(R^{p}, R^{q}\right)$. Then $K$ is called a model $G \times H$ singularity with respect to $\phi_{1}$ and $\phi_{2}$, if $K$ is invariant under the action of $G \times H$ on $\operatorname{Hom}\left(R^{p}, R^{q}\right)$.

Definition 1.2. Let $\xi=(\pi: E \rightarrow X)$ be a smooth vector bundle of fiber dimension $q$. Let $\pi_{E}: P_{E} \rightarrow X$ be the principal $\mathrm{GL}(q, R)$ bundle associated to $E$. Let $G$ be a Lie group. Let $\phi: G \rightarrow \mathrm{GL}(q, R)$ be a faithful representation of $G$. A $(G, \phi)$-structure on $E$ is given by a principal $G$-bundle $\pi_{G}: P_{G} \rightarrow X$, and a smooth mapping $\tilde{\phi}: P_{G} \rightarrow P_{E}$ such that

(a) $\pi_{E} \cdot \tilde{\phi}=\pi_{G}$,

(b) $\tilde{\phi}$ is smooth and $1-1$,

(c) for each $u \in P_{G}$ and $g \in G, \tilde{\phi}(u) \phi(g)=\tilde{\phi}(u g)$.

ReMARK. A $(G, \phi)$-structure on a vector bundle $\xi=(\pi: E \rightarrow X)$ of fiber dimension $q$, gives rise to a reduction of the structural group $\mathrm{GL}(q, R)$ to $\phi(G)$. Hence the representation $\phi$ allows us to consider the vector bundle $\xi$ as a vector bundle associated with the principal $G$-bundle $P_{G}$, because the $G$ action on the fibers will be linear.

1 This research was supported by the following contracts: NONR-266(57) and NSF-G-19022. It consists of part of the author's doctoral dissertation at Columbia University, 1963. 
Definition 1.3. Let $\pi: E \rightarrow X$ and $\pi^{\prime}: E^{\prime} \rightarrow Y$ be smooth vector bundles of fiber dimension $p$ and $q$ respectively. Let there be given a $\left(G, \phi_{1}\right)$-structure on $E$ and an $\left(H, \phi_{2}\right)$-structure on $E^{\prime}$. Let $K$ be a model $G \times H$ singularity with respect to the representations, $\phi_{1}$ and $\phi_{2}$. Let $\operatorname{Hom}\left(E, E^{\prime}\right)$ denote the vector bundle over $X \times Y$ defined in $\$ 2$ of [2]. Then the $\left(G, \phi_{1}\right)$-structure on $E$ and the $\left(H, \phi_{2}\right)$-structure on $E^{\prime}$ induce a $\left(G \times H, \phi_{1}^{t^{-1}} \times \phi_{2}\right)$-structure on $\operatorname{Hom}\left(E, E^{\prime}\right)$. Hence we can consider $\operatorname{Hom}\left(E, E^{\prime}\right)$ as a vector bundle with fiber $\operatorname{Hom}\left(R^{p}, R^{a}\right)$, structural group $G \times H$, and base $X \times Y$. Hence $K$ defines a regular manifold collection $K(X, Y)$, of submanifolds of $\operatorname{Hom}\left(E, E^{\prime}\right)$. Let $K=K_{1} \cup \ldots \cup K_{s}$, then $K(X, Y)=K_{1}(X, Y) \cup \cdots \cup K_{s}(X, Y)$, and the $\operatorname{codim}\left(K_{i}\right)=\operatorname{codim}\left(K_{i}(X, Y)\right) . K(X, Y)$ is called the $G \times H$ singularity defined by the $\left(G, \phi_{1}\right)$-structure on $E$, the $\left(H, \phi_{2}\right)$-structure on $E^{\prime}$, and the model $G \times H$ singularity $K$.

Definition 1.4. Let $\pi: E \rightarrow X$ and $\pi^{\prime}: E \rightarrow Y$ be smooth vector bundles. Let there be given a $\left(G, \phi_{1}\right)$-structure on $E$ and an $\left(H, \phi_{2}\right)$ structure on $E^{\prime}$. Let $K$ be a model $G \times H$ singularity, and let $K(X, Y)$ be the $G \times H$ singularity defined by the $G$-structure on $E$ the $H$ structure on $E^{\prime}$, and the $G \times H$ singularity $K$. Let $f: E \rightarrow E^{\prime}$ be a smooth vector bundle homomorphism covering $\bar{f}: X \rightarrow Y$. This induces a map $\hat{f}: X \rightarrow \operatorname{Hom}\left(E, E^{\prime}\right)$ defined by $\hat{f}(x)=f \mid E_{x} \in \operatorname{Hom}\left(E_{x}, E_{f(x)}^{\prime}\right)$, for $x \in X$. We say $x$ is a $K$-singular point of $f$ if $\hat{f}(x) \in K(X, Y)$. We say that $f$ displays the singularity transversally if $\hat{f}(X)$ meets $K(X, Y)$ transversally. The set $\hat{f}^{-1}(K(X, Y))=S_{K}(f)$ is called the set of $K$ singular points of $f$. If $f$ displays $K$ transversally then $S_{K}(f)$ is a manifold collection of the same codimension as $K(X, Y)$.

Let the coefficient ring for all characteristic classes be $Z_{2}$.

Definition 1.5. Let $\pi: E \rightarrow X$ be a smooth vector bundle. Let $\pi_{G}: P_{G} \rightarrow X$ be the principal $G$-bundle given by a $(G, \phi)$-structure on $E$. The characteristic classes of the $G$-structure $(G, \phi)$ on $E$ are defined to be the characteristic classes of $P_{G}$. If the maximal compact subgroup $G_{c}$ of $G$ is the orthogonal group $O(k)$, then the characteristic classes of the $G$-structure are just the Stiefel-Whitney classes of $P_{G}$. These will be called the Stiefel-Whitney classes associated with the $(G, \phi)$-structure on $E$. We will denote these classes by $W_{i}^{G}(E) \in H^{i}(X)$.

With these definitions we get the following generalizations of the theorem of Thom (see [6], [7]).

Theorem 1.6. (1) Let $\xi=(\pi: E \rightarrow X)$ and $\eta=\left(\pi^{\prime}: E^{\prime} \rightarrow Y\right)$ be smooth vector bundles of fiber dimension $p$ and $q$ respectively. Let $\xi$ be given a $\left(G, \phi_{1}\right)$-structure and $\eta$ an $\left(H, \phi_{2}\right)$-structure. Assume the maximal compact subgroups $G_{c}$ and $H_{c}$ are respectively $O(k)$ and $O(t)$. 
(2) Let $K=K_{1} \cup \ldots \cup K_{a}$ be a model $G \times H$ singularity of codimension s. Assume that $K$ carries a fundamental class, that $K$ is closed in $\operatorname{Hom}\left(R^{p}, R^{q}\right)$, and that $K_{1}$ is a regular submanifold. (These are all satisfied if $K$ is a real algebraic variety.)

(3) Let $f: E \rightarrow E^{\prime}$ be a smooth homomorphism of vector bundles covering $\bar{f}: X \rightarrow Y$. Assume that $f$ displays the singularity $K$ transversally.

Then $S_{K}(f)$, the manifold collection of $K$ singular points of $f$ carries a fundamental class $\left[S_{K}(f)\right]$. Let $i: S_{K}(f) \rightarrow X$ be the inclusion map and let $D_{X}$ be Poincaré duality in $X$. There exists a polynomial $P$, which is independent of $f$, such that

$$
D_{X} i_{*}\left[S_{K}(f)\right]=P\left(W_{j}^{G}(E), \bar{f}^{*} W_{j}^{H}\left(E^{\prime}\right)\right) .
$$

Corollary 1.7. If the $P_{H}$ of the $H$-structure in 1.6 is isomorphic to the trivial bundle, then the class $\left[S_{K}(f)\right]$ is independent of $\bar{f}$.

REMARK. (1) An exact analogue of 1.6 can be proven for arbitrary $G$ - and $H$-structures. However it is the less general theorem above that we apply in what follows.

(2) I. Porteous (see [1]) has calculated the polynomials of Theorem 1.6 in the following case. Let $n=\min (p, q)$, let $G=\mathrm{GL}(p, R)$, let $H=\mathrm{GL}(q, R)$, let $\phi_{1}=i d_{G}$ and let $\phi_{2}=i d_{H}$. Let us denote by $Z_{a}$ the model $G \times H$ singularity defined by

$$
Z_{a}=\left(\sigma \in \operatorname{Hom}\left(R^{p}, R^{a}\right) \mid \operatorname{rank} \sigma=n-a\right) .
$$

Then $\bar{Z}_{a}=\bigcup_{a \geqq a^{\prime}} Z_{a^{\prime}} . \bar{Z}_{a}$ is a real algebraic variety and therefore satisfies all the hypotheses of 1.6. Porteous calculates the universally defined polynomial for the singularity $Z_{a}(X, Y)$ under a slightly different transversality assumption. It is not hard to show that the transversality assumption of 1.6 is stronger than Porteous', and hence his formulas hold.

2. Applications to osculating maps. Let us preserve the notation of 1.6. Assume that $\operatorname{dim} X=n$ and that $\operatorname{dim} Y=N$. Let $\left(D^{(k)}, k=1,2, \cdots\right)$ be a sequence of $k$ th order symmetric linear connections on $Y$. Let us assume that $E=T_{p}(X)$ and that $E^{\prime}=T_{1}(Y)$. Let $G=J^{p}(n)$, the group of invertible $p$-jets of maps from $R^{n}$ into $R^{n}$ which send the origin into the origin. The differential structure on $X$ endows $T_{p}(X)$ with a canonical $J^{p}(n)$-structure. Let there be given some arbitrary $H$-structure on $T_{1}(Y)$. Let $K$ be a model $J^{p}(n) \times H$ singularity with respect to the given structures. Let $f: X \rightarrow Y$ be a smooth map. The vector bundle homomorphism that we are interested in studying is the $p$ th order osculating map $\nabla_{p} T_{p}(f): T_{p}(X) \rightarrow T_{1}(Y)$. 
The following proposition shows that condition 3 of Theorem 1.6 is not very restrictive in the case outlined above.

Proposition 2.1. Let $X$ and $Y$ be smooth manifolds of dimension $n$ and $N$ respectively. Let $\left(D^{(k)}, k=1,2 \ldots\right)$ be a sequence of $k t h$ order symmetric linear connections on $Y$. Let there be given an $H$-structure on $T_{1}(Y)$ and a $G$-structure on $T_{p}(X)$. Let $K$ be any model $G \times H$ singularity, and let $K(X, Y)$ be the corresponding singularity induced by the $G$ structure, the $H$-structure and $K$. Then the set of $f \in C(X, Y)$ such that $\left(\nabla_{p} T_{p}(f)\right)^{\wedge}(X)$ meets $K(X, Y)$ transversally is dense in $C(X, Y)$.

The remainder of this announcement will deal with the applications of 2.1 and 1.6 to some concrete problems of "affine" and "Riemannian" singularities.

3. Inflection points. We will fix the following notation for the next two sections. Let $X$ and $Y$ be smooth manifolds of dimension $n$ and $N$ respectively. Let $\left(D^{(k)}, k=1,2 \cdots\right)$ be a sequence of $k$ th order symmetric linear connections on $Y$. Let $\nu(n, p)$ denote the fiber dimension of $T_{p}(X)$. Assume that $N>\nu(n, p)$.

Definition 3.1. Let $f: X \rightarrow Y$ be a $p$ th order nondegenerate immersion of $X$ in $Y$, and let $N_{p, f, Y}(X)$ be the $p$ th order normal bundle of $X$ in $Y$ with respect to $f$.

Let $\nu_{p, f}: O^{p+1} T_{1}(X) \rightarrow N_{p, f, Y}(X)$ be the $p$ th order normal form of $f$. $x \in X$ is said to be a pth order inflection point of $f$ if $\nu_{p, f}$ is not of maximal rank on $O^{p+1} T_{1}(X)_{x}$.

Let $I^{p}(X, Y)$ denote the space of $p$ th order nondegenerate immersions of $X$ in $Y$. Let $S_{i}\left(\nu_{p, f}\right)=\left(x \in X \mid \operatorname{rank}\left(\nu_{p, f}\right)\right.$ on

$$
\left.O^{p+1} T_{1}(X)_{x}=\min (\nu(n, p+1)-\nu(n, p), N-\nu(n, p))-i\right) .
$$

Theorem 3.2. (a) There exists an open dense subset $D \subseteq I^{p}(X, Y)$ such that if $f \in D$ then the set of pth order inflection points of $f$ is a manifold collection $I_{f}=S_{1}\left(\nu_{p, f}\right) \cup \ldots \cup S_{k}\left(\nu_{p, f}\right)$ of submanifolds of $X$. Furthermore we have the following results.

(b) Let $N \geqq \nu(n, p+1)+n$ or let $\nu(n, p)<N \leqq \nu(n, p+1)-n$. If $f \in D$ then $I_{f}=\varnothing$ and hence $f$ is a $p+1$ order nondegenerate immersion.

(c) Let $\nu(n, p+1)-n<N<\nu(n, p+1)+n$. Let $f \in D$. $\left[S_{i}\left(\nu_{f, p}\right)\right]-$ $=S_{i}\left(\nu_{p, f}\right) \cup \ldots \cup S_{k}\left(\nu_{p, f}\right)$. Then $\left[S_{i}\left(\nu_{p, f}\right)\right]-$ carries a fundamental class $\left[S_{i}\left(\nu_{p, f}\right)\right]-$. Let $D_{X}$ be Poincaré duality in $X$ and let $j: S_{i}\left[\left(\nu_{p ; f}\right)\right]-$ $\subseteq X$ be the inclusion map. Then

$$
D_{x} j_{*}\left[S_{i}\left(\nu_{p, f}\right)\right]^{-}=P\left(W_{a}(X), f^{*} W_{b}(Y)\right),
$$

where $P$ is a universally defined polynomial whose definition is inde- 
pendent of $f$, and where $W_{a}(X)$ and $W_{b}(Y)$ are the Stiefel-Whitney classes of $X$ and $Y$ respectively.

Remark. The polynomial $P$ can be computed from Porteous' formulas, Whitney duality, and the formulas for the Whitney classes of the tensor product of vector bundles.

The following examples are easy consequences of the above theorems.

Proposition 3.3. Let $S^{1}$ be the circle. Let $Y$ be unorientable. Let $f \in I^{N-1}\left(S^{1}, Y\right)$. Assume that $f^{*} W_{1}(Y) \neq 0$. Then $f$ has an $(N-1)$ st order inflection point. If $N=2$ this tells us that any immersion $f$ of the circle in an unorientable two manifold has an inflection point if $f^{*} W_{1}(Y) \neq 0$.

Proposition 3.4. Let $\operatorname{dim} X=2$, and let us be concerned with first order inflection points. We will endow the target manifold $Y$ with an arbitrary symmetric connection.

(a) Let $X=P_{2}(R)$ and let $Y=P_{6}(R)$. Let $f: P_{2}(R) \rightarrow P_{6}(R)$ be the natural embedding of $P_{2}$ as a projective plane. Then any immersion of $P_{2}(R) \rightarrow P_{6}(R)$ homotopic to $f$ has inflection points. The same fact is true for the natural embedding of $P_{2}$ in $P_{4}$.

Proposition 3.5. Let $X=P_{2}(C)$. We will again be concerned with first order inflection points. We again endow the target manifold $Y$ with an arbitrary symmetric linear connection.

(a) If $Y=R^{17}$ (in fact any 17 manifold $Y$ with $W_{1}(Y)=0$ will suffice), then any immersion of $P_{\mathbf{2}}(C)$ in $Y$ must have inflection points.

(b) If $Y=R^{14}$, then for any immersion of $P_{2}(C)$ in $R^{14}$, there exists $a$ point $x \in P_{2}(C)$ such that $\operatorname{dim}\left(\operatorname{ker}\left(\nu_{1, f}(x)\right) \geqq 2\right.$.

Many more examples of this type can be given so we will stop here.

\section{Totally geodesic points.}

Definition 4.1. Let $N>\nu(n, p)$, and let $f: X \rightarrow Y$ be a $p$ th order nondegenerate immersion. Let $N_{p, f, Y}(X)$ be the $p$ th order normal bundle of $X$ in $Y$ with respect to $f$, and let $\nu_{p, f}: O^{p+1} T_{1}(X) \rightarrow N_{p, f, Y}(X)$ be the $p$ th order normal form. $x \in X$ is said to be a pth order totally geodesic point with respect to the connections of the immersion $f$, if $\nu_{p, f}$ is the zero map on the fiber $O^{p+1} T_{1}(X)_{x}$. If this true at all the $x \in X, f$ is called a pth order totally geodesic immersion.

REMARK. In the classical case $p=1$ this definition is well known to be equivalent to the usual definition.

Proposition 4.2. Let $N>\nu(n, p)$, and let $\operatorname{dim} X \geqq 2$. Let $I^{p}(X, Y)$ 
be the set of pth order nondegenerate immersions of $X$ in $Y$. Then the set of $f \in I^{p}(X, Y)$ without pth order totally geodesic points is open and dense in $I^{p}(X, Y)$.

5. Parabolic points. We will adopt the following notation for the next two sections. $X$ will denote an $n$-dimensional manifold. Let $Y=R^{n+1}$ and endow $Y$ with its usual Riemann (Euclidean) metric $\langle$,$\rangle . Let I\left(X, R^{n+1}\right)$ denote the space of immersions of $X$ in $R^{n+1}$. If $f \in I\left(X, R^{n+1}\right)$ denote by $w_{2} T_{2}(f): T_{2}(X) \rightarrow T_{1}\left(R^{n+1}\right)$ the second order osculating map of $f$ with respect to the usual connection on $R^{n+1}$. Denote by $\left(w_{2} T_{2}\right) \wedge(f): X \rightarrow \operatorname{Hom}\left(T_{2}(X), T_{1}(Y)\right)$ the section of Hom $\left(T_{2}(X), T_{1}\left(R^{n+1}\right)\right)$ over the graph of $f$, determined by the homomorphism $w_{2} T_{2}(f)$.

Definition 5.1. Let $f \in I\left(X, R^{n+1}\right)$. Let $N_{f}(X)$ and $\nu_{f}: O^{2} T_{1}(X)$ $\rightarrow N_{f}(X)$ be the normal bundle and the normal form of the immersion $f$ respectively. Let $x \in X$ and $U$ be a neighborhood of $x$ in which we can define a unit normal vector field $N$ to $f(U)$. For every point $x^{\prime} \in U$ we can define a self adjoint linear transformation $S_{N\left(x^{\prime}\right)}$ : $T_{1}(X)_{x^{\prime}} \rightarrow T_{1}(X)_{x^{\prime}}$ defined by the formula

$$
\left\langle S_{N\left(x^{\prime}\right)} u, v\right\rangle=\left\langle v_{f}(u, v), N\left(x^{\prime}\right)\right\rangle, \quad u, v \in T_{1}(X)_{x^{\prime}} .
$$

Let $K_{N, f}\left(x^{\prime}\right)=\operatorname{Det}\left(S_{N\left(x^{\prime}\right)}\right) . K_{N, f}\left(x^{\prime}\right)$ is called the Gauss-Kronecker curvature of $f$ at $x^{\prime}$ with respect to the normal direction $N\left(x^{\prime}\right)$.

DEFINITION 5.2. $x^{\prime} \in X$ is called a parabolic point of the immersion $f$ if $K_{N, f}\left(x^{\prime}\right)=0$. This is clearly independent of the unit normal chosen, and depends only upon the immersion $f$ and the Riemann structure on $R^{n+1}$.

Proposition 5.3. Let $f \in I\left(X, R^{n+1}\right)$. Let $G_{N}(f): X \rightarrow P_{n}(R)$ be the Gauss normal map of $f . x$ is a parabolic point of $f$ if and only if $x$ is not a regular point of $G_{N}(f)$.

THEOREM 5.4. There exists a model $J^{2}(n) \times O(n+1)$ singularity $P \subset \operatorname{Hom}\left(R^{\nu(n, p)}, R^{n+1}\right)$ with the following properties.

(a) $P$ is a real algebraic variety of codimension 1 .

(b) Let $P\left(X, R^{n+1}\right)$ be the singularity induced by the canonical $J^{2}(n)$ structure on $T_{2}(X)$, the Riemann structure on $R^{n+1}$, and the model singularity $P$. Then $x \in X$ is a parabolic point if and only if $\left(w_{2} T_{2}\right)^{\wedge}(f)(x)$ $\in P\left(X, R^{n+1}\right)$.

(c) Let $D_{p}=\left(f \in I\left(X, R^{n+1}\right) \mid\left(w_{2} T_{2}\right)^{\wedge}(f)\right.$ take the singularity $P\left(X, R^{n+1}\right)$ transversally). The elements of $D_{p}$ are called parabolic generic immersions. The parabolic generic immersions are open and dense in $I\left(X, R^{n+1}\right)$. 
Theorem 5.5. Let $f \in I\left(X, R^{n+1}\right)$. Let $S_{p}(f)$ be the set of parabolic points of $f$, let $i: S_{p}(f) \rightarrow X$ be the inclusion map, and let $D_{X}$ be Poincaré duality in $X$. If $f \in D_{q}$ then $S_{p}(f)$ is a manifold collection and carries a fundamental class $\left[S_{p}(f)\right]$. Furthermore we have the formula, $D_{X} i_{*}\left[S_{P}(f)\right]$ $=p(n) W_{1}(X)$, where $p(n)$ is a $Z_{2}$ valued function depending on the dimension $n$ of $X$ alone.

5.5 and a deeper analysis of the singular variety yield the following corollaries.

Corollary 5.6. (a) Let $X$ be a compact orientable $n$-manifold. Let $f \in D_{P}$. Then the set of parabolic points of $f$ is homologous to zero.

(b) Let $X$ be a compact 2-manifold. Let $f \in D_{P}$. Then the set of parabolic points $S_{P}(f)$ is a set of disjoint circles which when considered as a homology class are homologous to zero.

(c) Let $X$ be an odd dimensional, compact, unorientable manifold. Then any immersion of $X$ in $R^{n+1}$ has parabolic points.

\section{Umbilic points.}

Definition 6.1. Let $f \in I\left(X, R^{n+1}\right)$. Let $x \in X$ and let $U$ be a sufficiently small neighborhood of $x$ so that we can define a unit vector field $N$ normal to $f(U)$. Let $S_{N(x)}: T_{1}(X)_{x} \rightarrow T_{1}(X)_{x}$ be the self adjoint linear transformation defined in 5.1. The eigenvalues $k_{i}(x)$ of this operator are called the principal curvatures of $f$ at $x$. These numbers are uniquely determined up to sign in the sense that if we picked $-N$ instead of $N$ we would merely change the sign of $k_{i}(x)$.

Definition 6.2. Let $f \in I\left(X, R^{n+1}\right)$. A point $x \in X$ is called an umbilic point of the immersion $f$ if all the principal curvatures of $f$ at $x$ are equal. It is clear that the property of being an umbilic point is independent of the unit normal chosen.

THEOREM 6.3. There exists a model $J^{2}(n) \times O(n+1)$ singularity $U \subset \operatorname{Hom}\left(R^{\nu(n, 2)}, R^{n+1}\right)$ with the following properties.

(a) $U$ is a real algebraic variety of codimension

$$
\frac{n(n+1)}{2}-1
$$

(b) Let $U\left(X, R^{n+1}\right)$ be the singularity induced by the $J^{2}(n)$-structure on $T_{2}(x)$ the Riemann structure on $R^{n+1}$, and the model singularity $U$. Then $x \in X$ is an umbilic point if and only if $\left(w_{2} T_{2}\right)^{\wedge}(f) \in U\left(X, R^{n+1}\right)$.

Theorem 6.4. Let $\operatorname{dim} X \geqq 3$. Then the set of $f \in I\left(X, R^{n+1}\right)$ such that $f$ has no umbilic points is open and dense in $I\left(X, R^{n+1}\right)$. 
Theorem 6.5. Let $X$ be a compact orientable 2-manifold. Then there exists an open dense subset $D_{U} \subseteq I\left(X, R^{3}\right)$ with the following properties. If $f \in D_{U}$ then

(a) the set of umbilic points $S_{U}(f)$ is a finite set of points, and

(b) (the number of umbilic points) $\bmod 2=0$.

Corollary 6.6. Let $X$ be a compact orientable 2-manifold. Assume that the Euler characteristic $\chi(X) \neq 0$. Let $D_{U} \subset I\left(X, R^{3}\right)$ be the open dense subset of 6.5. If $f \in D_{U}$, then $f$ has at least two umbilic points.

REMARK. This last corollary gives a partial answer and generalization to a conjecture of $\mathrm{C}$. Carathéodory. Carathéodory conjectured that any convex embedding of $S^{2}$ in $R^{3}$ has at least two umbilic points. In the case where the embedding is real analytic the conjecture has been settled in the affirmative, however the $C^{k}$ case $(k \leqq \infty)$ is still an open problem.

Added in proof. Theorem 6.5 can be strengthened to the following theorem.

Theorem 6.7. Let $X$ be a compact 2-manifold. Then there exists an open dense subset $D_{U} \subseteq I\left(X, R^{3}\right)$ with the following properties. If $f \in D_{U}$ then,

(i) the set of umbilic points of $f$ is a finite subset of $X$, and

(ii) the index of each of these umbilic points is $\pm \frac{1}{2}$.

Hence we have that (the number of umbilic points of $f) \geqq 2|\chi(X)|$, where $\chi(X)$ is the Euler characteristic.

\section{REFERENCES}

1. I. R. Porteous, Simple singularities of maps (Mimeographed notes), Columbia University, New York, 1962.

2. E. A. Feldman, The geometry of immersions. I, Bull. Amer. Math. Soc. 69 (1963), 693-698.

3. R. Thom (notes by H. Levine), Singularities of differentiable mappings (Mimeographed notes), Bonn University, Bonn, 1959.

4. $\mathrm{H}$. Whitney, On singularities of mappings of Euclidean spaces. I. Mappings of the plane into the plane, Ann. of Math (2) 62 (1955), 347-410.

5. - Elementary structure of real algebraic varieties, Ann. of Math. (2) 66 (1957), 545-556.

6. André Haefliger, Les singularités des applications différentiables, Séminaire Henri Cartan (1956/57), Exposé 7, Secrétariat mathématique, Paris, 1958.

7. André Haefliger and A. Kosinski, Un théorème de Thom sur les singularités des applications différentiables, Séminaire Henri Cartan (1956/57), Exposé 8, Secrétariat mathématique, Paris, 1958.

Princeton University 